\title{
Clostridium cadaveris bacteremia in a critically ill surgical patient with septic shock after total abdominal colectomy:
} a case report and review of literature

\author{
Pfaff $\mathrm{CE}^{1 *}$, Droege $\mathrm{CA}^{2,3}$, Magner $\mathrm{ME}^{4}$ and Schrager $\mathrm{JJ}^{5,6}$ \\ ${ }^{1}$ Department of Pharmacy Services, UC Health - West Chester Hospital, West Chester Township, Ohio, USA \\ ${ }^{2}$ Department of Pharmacy Services, UC Health - University of Cincinnati Medical Center Cincinnati, Ohio, USA \\ ${ }^{3}$ University of Cincinnati James L. Winkle College of Pharmacy, Cincinnati, Ohio, USA \\ ${ }^{4}$ Department of Neurosurgery, The Christ Hospital Physicians, Cincinnati, Ohio,USA \\ ${ }^{5}$ Department of Surgery - Division of Trauma, University of Cincinnati, Cincinnati, Ohio, USA \\ ${ }^{6}$ University of Cincinnati College of Medicine, Cincinnati, Ohio, USA
}

\begin{abstract}
Sepsis secondary to bacteremia remains highly prevalent amongst critically ill patients. Aerobic Gram positive and negative bacterial infections remain the most common sources while fungal and anaerobic pathogens are comparatively infrequent. Broad empiric antimicrobial therapy may not specifically target nonpathogenic anaerobic bacteria with unique resistance patterns. Clostridium cadaveris is an anaerobic Gram-positive bacillus commonly found in the gastrointestinal tract of humans and other mammals. This species of clostridia is rarely responsible for infectious pathology in living patients. Nine case reports of $C$. cadaveris infection have been published to date. We describe a 41-year-old female admitted to our surgical intensive care unit after total abdominal colectomy for severe Crohn's disease. Post-operatively an intra-abdominal hematoma was identified on imaging and blood cultures were positive for concomitant C. cadaveris and Parvimonas micra bacteremia. Infections were successfully cleared with broad spectrum antibiotics including intravenous meropenem and metronidazole. The patient progressed into multi-system organ failure and expired on post-operative day 44 . This case reflects the observed high mortality rate in the limited published literature regarding $C$. cadaveris bacteremia. The clinician must be vigilant in obtaining cultures, source control and appropriate empiric and targeted antimicrobial therapy in the critically ill surgical patient.
\end{abstract}

\section{Introduction}

Bacteremia and resultant sepsis have undergone many definition changes over the centuries including the process by which flesh rots, to "blood poisoning", to the modern designation of systemic inflammatory response syndrome in reaction to active infection [13]. Most recent consensus have designated "severe sepsis" and "septic shock" to describe infection syndromes complicated by acute organ dysfunction $[4,5]$.

A point prevalence study of 13,796 adult patients performed in 2007 revealed that culture-positive bloodstreams were responsible for $15.1 \%$ of all infections seen in the intensive care unit [6]. This prevalence increased to $25.9 \%$ in North America intensive care units. Gram negative and positive isolates served as the two most common pathogen categories at 59.8 and 51\% respectively. Anaerobic bacteremia was appreciated in $7.9 \%$ of North American patients and only $4.5 \%$ worldwide [6].

Clostridium cadaveris is an anaerobic Gram-positive bacillus commonly found in the gastrointestinal tract of humans and other mammals [7]. This non-toxin producing clostridia species was first described by Klein and colleagues in 1899 when it was identified as the microbial species responsible for gaseous production during human decomposition [8]. Although present in the intestinal flora of humans, C. cadaveris is rarely responsible for infectious pathology in living patients [7]. Nine case reports of C. cadaveris infection have been published to date [9-15].

\section{Case report}

A 41-year-old, morbidly obese female with past medical history significant for ulcerative colitis presented to the emergency department with complaints of abdominal pain, tachycardia and hypotension. The patient reported a three month history of eight to ten bloody, loose stools daily and was diagnosed with acute deep vein thrombosis one week prior to admission. Warfarin and enoxaparin anticoagulation therapies were initiated at that time. Computed tomography scan on admission identified new pulmonary embolus for which an inferior vena cava filter was placed in light of the presence of hematochezia. Methylprednisolone therapy was administered for four days to treat an ulcerative colitis flare.

Hemodynamic response was noted to blood transfusions and volume resuscitation. The abdominal symptoms and hematochezia

Correspondence to: Caitlin Pfaff, PharmD, BCPS, Department of Pharmacy Services, UC Health - West Chester Hospital, 7700 University Drive, West Chester Township, OH 45069, USA

Key words: Clostridium cadaveris, bacteremia, sepsis, critical care, surgery

Received: December 04, 2016; Accepted: December 22, 2016; Published: December 26, 2016 
did not resolve despite an aggressive steroid regimen. On hospital day ten imaging studies showed a progressively more dilated colon and laboratory studies revealed a white blood cell count of 49,000 cells per microliter. The patient underwent total abdominal colectomy and end ileostomy on hospital day 11 with return of gastrointestinal function with ileostomy output on post-operative day two. On post-operative day eight, she became tachycardic and oliguric and was transferred to the surgical intensive care unit. Surveillance blood cultures were obtained at this time. Computed tomography scan of the abdomen and pelvis showed free fluid in the abdomen, possibly some hematoma, no abscess collection, and no leak from the residual rectal stump.

Despite resuscitation and empiric coverage with piperacillintazobactam, her clinical condition to septic shock and she required emergent intubation on post-operative day ten and experienced a witnessed aspiration during the procedure. Multi-system organ failure developed requiring prone ventilation and inhaled nitric oxide for severe acute respiratory distress syndrome, continuous renal replacement therapy for acute kidney injury, and norepinephrine and vasopressin for hemodynamic instability. She manifested a significant coagulopathy with profound synthetic liver abnormalities (total bilirubin peaked at 10.6 milligrams per deciliter and peak serum ammonia concentration of 180 micrograms per deciliter), thrombocytopenia with a nadir of nine thousand cells per microliter, and altered mental status. Heparininduced thrombocytopenia panels were negative. Surveillance blood cultures were positive for Parvimonas micra from a peripherally inserted central catheter line and Clostridium cadaveris returned from a peripheral culture. Broncheoalveolar lavage at time of intubation demonstrated 10,000-100,000 colony forming units per milliliter of Candida albicans. Cultures sent from the abdominal fluid collection drainage on post-operative day 13 revealed vancomycin-sensitive Enterococcus faecalis, vancomycin-resistant Enterococcus faecium and pan-sensitive Klebsiella pneumoniae. The antibiotic regimen was broadened to include intravenous linezolid, meropenem, metronidazole and fluconazole for a two-week course to account for known and anticipated resistance patterns. An intensive care unit pharmacist was present on multidisciplinary rounds to facilitate monitoring, adjustment and optimization of pharmacokineticallydriven dosing and pharmacodynamic outcomes.

Though critically ill, the patient's condition gradually improved. Vasoactive agents were weaned off, acute kidney injury resolved, and the patient became more responsive to stimuli. Ventilator support was weaned though she did undergo tracheostomy on postoperative day 35. Blood cultures were negative on post-operative day 27 and 37. The coagulopathy resolved despite continued abnormal synthetic liver function. She was able to tolerate goal nutrition and her prealbumin increased to 15.9 milligrams per deciliter from its nadir of 4.9 milligrams per deciliter. Despite improvement, she began to manifest signs of sepsis on post-operative day 40 with a febrile response and hypotension. Broad spectrum antimicrobial therapy was initiated to cover for previously-identified organisms. Culture results positively demonstrated Proteus penneri in the urine. Despite appropriate antimicrobial coverage and resuscitation, septic shock and multi-system organ failure progressed. The morning of post-operative day 44, hospital day 55 the patient experienced pulseless electrical activity arrest and received three rounds of advanced cardiovascular life support protocol for a total of seven minutes with return of spontaneous circulation. Vasopressor requirements increased and hypoxia worsened despite aggressive ventilatory support. At this time family elected to pursue comfort measures and the patient rapidly progressed to asystole.

\section{Discussion}

The following literature review was completed using the PubMed database. Search terms included "Clostridium cadaveris", "bacteremia", "infection" and "anaerobic bacterial infection". Identified articles were reviewed by at least two authors and are summarized below.

The first case report of $C$. cadaveris infection was published in 1992 when Herman and colleagues described a 58-year-old female with past medical history significant for alcohol abuse who presented with jaundice, abdominal distension, tenderness and ascites. Initial ascitic fluid cultures were negative and she received a 14-day course

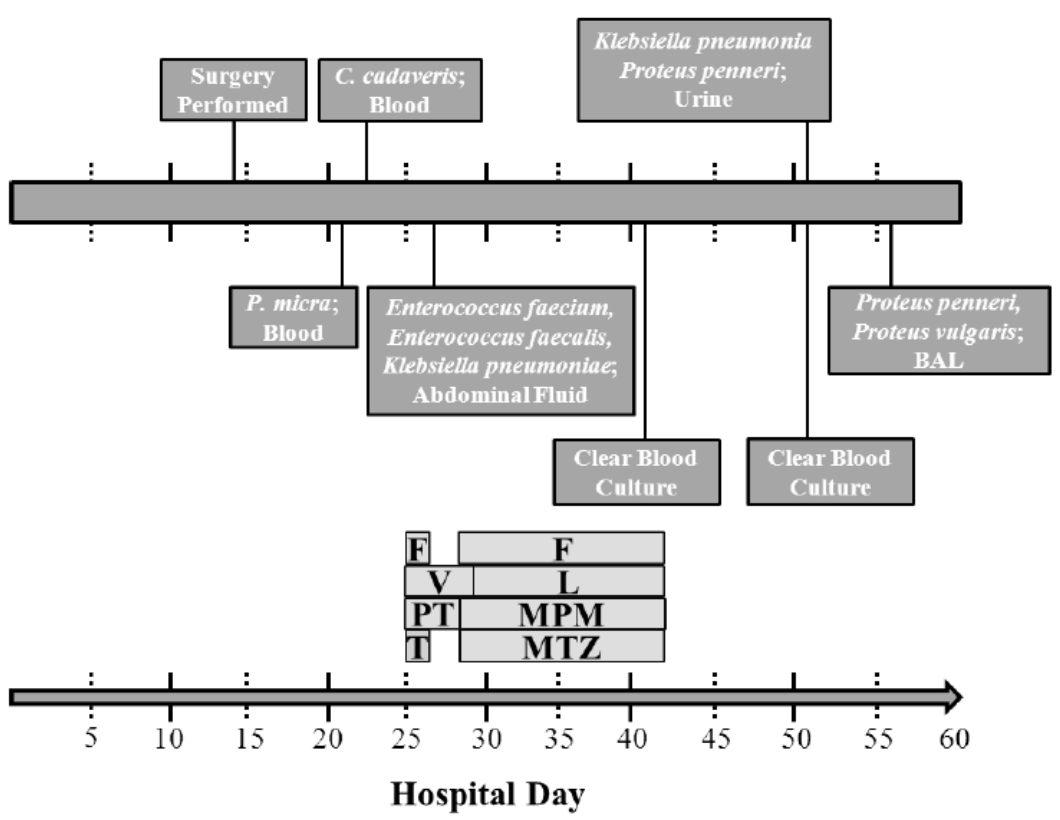

Figure 1. Culture and Antimicrobial Timeline. (PT: piperacillin-tazobactam; T: tobramycin; V: vancomycin; F: fluconazole; L: linezolid; MPM: meropenem; MTZ: metronidazole). 
Pfaff CE (2016) Clostridium cadaveris bacteremia in a critically ill surgical patient with septic shock after total abdominal colectomy: a case report and review of literature

Table 1. Summary known of C. cadaveris cases. (NR: Not reported).

\begin{tabular}{|c|c|c|c|c|c|c|c|}
\hline Year Reported & Age & Sex & Presentation & $\begin{array}{c}\text { Source } \\
\text { Identified }\end{array}$ & Antibiotics & Sensitivities & Expired \\
\hline $1992[3]$ & 58 & $\mathrm{~F}$ & $\begin{array}{l}\text { Abdominal distension, nausea, vomiting } \\
\text { and jaundice }\end{array}$ & Ascitic fluid & $\begin{array}{l}\text { Sulfamethoxazole-Trimethoprim } \\
\text { Imipenem- cilastatin } \\
\text { Cefotetan, aztreonam and penicillin G }\end{array}$ & NR & $\mathrm{Y}$ \\
\hline $1993[4]$ & 61 & $\mathrm{~F}$ & Fever, back pain and leukocytosis & Blood & $\begin{array}{l}\text { Imipenem-cilastatin, amikacin and } \\
\text { vancomycin }\end{array}$ & NR & $\mathrm{Y}$ \\
\hline $1993[4]$ & 66 & $\mathrm{~F}$ & Fever, cough and splenomegaly & Blood & $\begin{array}{l}\text { Mezlocillin and gentamicin } \\
\text { Changed to metronidazole }\end{array}$ & NR & $\mathrm{Y}$ \\
\hline 1997 [5] & 33 & M & Fever, leukocytosis and pleural effusion & Pleural fluid & $\begin{array}{l}\text { Amoxicillin-clavulanate } \\
\text { Changed to metronidazole }\end{array}$ & $\begin{array}{l}\text { Amoxicillin-clavulanate, } \\
\text { metronidazole }\end{array}$ & $\mathrm{N}$ \\
\hline $1999[6]$ & 42 & M & $\begin{array}{l}\text { Fever, hypotension and necrotic } \\
\text { decubitus ulcers }\end{array}$ & Blood & Ticarcillin-clavulanate and gentamicin & $\begin{array}{l}\text { Ampicillin-sulbactam, } \\
\text { chloramphenicol, ticarcillin- } \\
\text { clavulanate, cefotetan, } \\
\text { cefotaxime, clindamycin, } \\
\text { pencillin }\end{array}$ & $\mathrm{N}$ \\
\hline $2006[7]$ & 75 & M & $\begin{array}{l}\text { Fever, dyspnea, abdominal pain and } \\
\text { perforated diverticulitis }\end{array}$ & Blood & $\begin{array}{c}\text { Cefotaxime } \\
\text { Changed to amoxicillin, ciprofloxacin } \\
\text { and metronidazole }\end{array}$ & Metronidazole & $\mathrm{N}$ \\
\hline $2006[7]$ & 68 & M & Fever and abdominal pain & Blood & $\begin{array}{l}\text { Cefazolin and ciprofloxacin } \\
\text { Changed to amoxicillin-clavulanate } \\
\text { Changed to metronidazole }\end{array}$ & Clindamycin, metronidazole & $\mathrm{Y}$ \\
\hline $2007[8]$ & 60 & $\mathrm{~F}$ & Hip pain and fever & Hip joint & $\begin{array}{l}\text { Pencillin } \mathrm{G} \text { and cefazolin } \\
\text { Changed to clindamycin }\end{array}$ & NR & $\mathrm{N}$ \\
\hline 2009 [9] & 79 & $\mathrm{~F}$ & $\begin{array}{l}\text { Abdominal pain, diarrhea, left lower } \\
\text { quadrant mass }\end{array}$ & $\begin{array}{l}\text { Abdominal } \\
\text { abscess }\end{array}$ & $\begin{array}{l}\text { Piperacillin-tazobactam } \\
\text { Changed to metronidazole }\end{array}$ & NR & $\mathrm{N}$ \\
\hline
\end{tabular}

of broad spectrum antibiotics prior to discharge. The patient was readmitted with fever and altered mental status four days later and expired on hospital day 28. Post-mortem ascitic fluid cultures were positive for $C$. cadaveris. It is possible the translocation of $C$. cadaveris from the intestinal tract into the ascitic fluid was propagated by bowel perforation during one of the paracenteses the patient underwent during both hospital admissions. C. cadaveris strain antimicrobial sensitivities were not reported.

Gucalp and colleagues published two case reports detailing $C$. cadaveris bacteremia in immunocompromised patients. The first patient, a 61-year-old female, presented with renal cell carcinoma which extended into the lesser colon with resultant abscess formation. The patient underwent a radical nephrectomy, splenectomy and splenic flexure colon resection complicated by septic shock necessitating vasopressor support. The patient expired secondary to myocardial infarction on hospital day 40 . The second patient, a 66-year-old female, presented with proliferative lymphoblastic disease three months after surgical removal of colonic polyps. Initial blood cultures grew C. cadaveris which was treated with intravenous metronidazole. The patient expired secondary to multi-system organ failure. Each patient had possible mechanisms for gut translocation of $C$. cadaveris: tumor extension into the colon or surgical removal of colonic polyps.

Stolk-Engelaar and colleagues described a case of a 33-yearold male pig farmer with a pleuropulmonary empyema due to $C$. cadaveris and $C$. difficile after surgical removal of a bronchogenic cyst. C. cadaveris continued to grow in body fluid cultures for four weeks. The patient received amoxicillin-clavulanate and metronidazole with successful treatment of infection after empyema drainage.

Poduval and colleagues described C. cadaveris bacteremia in a 42 -year-old male with paraplegia secondary to a previous gunshot wound who presented from a nursing facility. The patient was found to have multiple necrotic decubitus ulcers, fever and hypotension. Initial blood cultures were positive for $C$. cadaveris sensitive to ampicillinsulbactam, chloramphenicol, cefotetan, clindamycin and penicillin. A bone scan was suggestive of left calcaneal osteomyelitis. The patient responded to antibiotic treatment and follow-up cultures were clear of pathogens.

Shade and colleagues reported two cases of $C$. cadaveris bacteremia. The first patient, a 75-year-old-male, presented with fever and dyspnea. Abdominal ultrasound revealed free air and the patient underwent laparotomy and subsequent sigmoidectomy for bowel perforation. Blood cultures from the operative day yielded C. cadaveris sensitive to metronidazole. The patient fully recovered and was discharged home after ten days of antibiotic therapy. The second case was a 68 -yearold male who presented with fever and abdominal pain. Abdominal imaging was negative and cultures drawn on admission grew $C$. cadaveris sensitive to metronidazole. The patient was discharged home after 32 days of antibiotic therapy only to be readmitted four days later with sepsis. Blood cultures once again grew C. cadaveris sensitive to metronidazole. The patient expired after cardiopulmonary arrest. Postmortem examination revealed a culture negative pericardial abscess. The authors postulated the cultures were negative due to prolonged antibiotic exposure.

Morshed and colleagues reported C. cadaveris bacteremia in a 60 -year-old female with septic arthritis after total hip arthroplasty. The patient was treated with extensive wound debridement and six weeks of intravenous clindamycin therapy followed by lifelong oral clindamycin prophylaxis. The joint remained clear of infection for the 18-month duration before the patient expired.

Leung and colleagues reported C. cadaveris in a 79-year-old female after abdominal surgery for incarcerated left inguinal hernia repair followed by laparotomy for small bowel obstruction. An enterotomy was identified and repaired intra-operatively. She later presented from home with persistent diarrhea and abdominal pain with a palpable mass noted in the left lower quadrant. Abdominal computed tomography revealed a 17x19x9 centimeter intraperitoneal abscess. The abscess was drained and cultures revealed C. cadaveris in three separate bottles. The patient's condition improved with abscess drainage and antibiotics 
were continued for approximately two weeks.

This case report exhibited a similar presentation to the reviewed cases. The majority of cases involve a surgical procedure resulting in gut flora translocation to the peritoneal cavity and - in some cases the bloodstream. In the present case, $C$. cadaveris was cleared from the bloodstream while receiving intravenous metronidazole and meropenem. Antimicrobial sensitivities were not readily available.

Three of five patients with C. cadaveris bacteremia expired despite appropriate antimicrobial therapy in this review. The present case described C. cadaveris bacteremia in septic shock with patient expiration after culture clearance with aggressive broad spectrum antimicrobial therapy. It is unlikely C. cadaveris bacteremia was instrumental in the expiration of this patient. However, the observed high mortality rate associated with C. cadaveris bacteremia in published literature is reflected in the outcome of this case.

The present case was unique as it involved $C$. cadaveris bacteremia with concomitant $P$. micra bacteremia. It is noteworthy that the $P$. micra grew in blood cultures while the patient was receiving piperacillintazobactam; a penicillin antibiotic with anaerobic coverage. Resistance to penicillin and metronidazole has been documented previously [16]. Parvimonas micra, previously known as Peptostreptococcus micros and Micromonas micros, is an anaerobic Gram-positive coccus commonly found in the normal flora of the human mouth. This organism has been documented as the source of oral infections and also as a systemic pathogen in the literature [17]. Aspiration during intubation and subsequent development of aspiration pneumonitis and hospital acquired pneumonia is a possible method of translocation into the bloodstream. There are no documented reports of simultaneous $C$. cadaveris and P. micra bacteremia in the literature.

\section{Conclusion}

Clostridium cadaveris is a rare pathogen with only nine reported cases in the English literature since first reported in 1992. This is the first reported case of concomitant $C$. cadaveris and $P$. micra bacteremia with septic shock in a relatively immunocompetent patient. Despite a past medical history of ulcerative colitis, the patient was not routinely taking any scheduled pharmacologic immunosuppression for over six weeks. Additionally, she was able to develop an appropriate inflammatory response and leukocytosis to her infection and surgical procedure early in her admission. A surgical procedure leading to gut translocation is the most consistent mechanism for $C$. cadaveris pathogenesis reported among these cases.

These infections are associated with high mortality; however, causation should not be presumed given the illness severity and multiple comorbidities observed in the majority of reported cases. In the present case, the patient expired secondary to MSOF and overwhelming sepsis despite clearance of $C$. cadaveris and P. micra on culture samples. The clinician must be vigilant in obtaining cultures, source control and appropriate empiric and targeted antimicrobial therapy in the critically ill surgical patient. A multidisciplinary approach should optimize infection risk assessment and expedite initiation of appropriate empiric antimicrobial therapy in critically ill patients with severe sepsis or septic shock secondary to uncommon pathogens.

\section{References}

1. Majno G (1991) The ancient riddle of sigma eta psi iota sigma (sepsis). J Infect Dis 163: 937-945. [Crossref]

2. Funk DJ, Parrillo JE, Kumar A (2009) Sepsis and septic shock: a history. Crit Care Clin 25: 83-101, viii. [Crossref]

3. Bone RC, Sibbald WJ, Sprung CL (1992) The ACCP-SCCM consensus conference on sepsis and organ failure. Chest 101: 1481-1483. [Crossref]

4. Dellinger RP, Levy MM, Rhodes A, Annane D, Gerlach H, et al. (2013) Surviving sepsis campaign: international guidelines for management of severe sepsis and septic shock: 2012. Crit Care Med 41: 580-637. [Crossref]

5. Angus DC, van der Poll T (2013) Severe sepsis and septic shock. N Engl J Med 369 840-851. [Crossref]

6. Vincent JL, Rello J, Marshall J, Silva E, Anzueto A, et al. (2009) International study of the prevalence and outcomes of infection in intensive care units. JAMA 302: 2323 2329. [Crossref]

7. Willis AT (1977) Characteristics of pathogenic and related clostridia. In: Anaerobic Bacteriology: Clinical and Laboratory Practice. Butterworth Boston: 111-66.

8. Klein E (1899) Ein beitrag zur akteriologie der leichenverwesung. Zentralblat fur Bakteriologie, Parasitenkunde. Infektionskrankheiten und Hygiene 1: 278-84

9. Herman R, Goldman IS, Bronzo R, McKinley MJ (1992) Clostridium cadaveris: an unusual cause of spontaneous bacterial peritonitis. Am J Gastroenterol 87: 140-142. [crossref]

10. Gucalp R, Motyl M, Carlisle P, Dutcher J, Fuks J, et al. (1993) Clostridium cadaveris bacteremia in the immunocompromised host. Med Pediatr Oncol 21: 70-72. [Crossref]

11. Stolk-Engelaar V, Verwiel J, Bongaerts G, Linsen V, Lacquet L, et al. (1997) Pleural empyema due to Clostridium difficile and Clostridium cadaveris. Clin Infect Dis 25: 160. [Crossref]

12. Poduval RD, Mohandas R, Unnikrishnan D, Corpuz M (1999) Clostridium cadaveris bacteremia in an immunocompetent host. Clin Infect Dis 29: 1354-1355. [Crossref]

13. Schade RP, Van Rijn M, Timmers HJ, Dofferhoff AS, Klaassen CH, et al. (2006) Clostridium cadaveris bacteraemia: two cases and review. Scand J Infect Dis 38: 59-62. [Crossref]

14. Morshed S, Malek F, Silverstein RM, O’Donnell RJ (2007) Clostridium cadaveris septic arthritis after total hip arthroplasty in a metastatic breast cancer patient. $J$ Arthroplasty 22: 289-92.

15. Leung J, Sasson M, Patel SR, Viveiros K (2009) Clostrium cadaveris intra-peritoneal abscess. Am J Gastroenterol 104: 2635-2636. [Crossref].

16. Veloo ACM, Welling GW, Degener JE (2011) Antimicrobial susceptibility of clinically relevant gram-positive anaerobic cocci collected over a three-year period in the Netherlands. Antimicrob Agents Chemother 55: 1199-1203.

17. Sousa ELR, Gomes BPFA, Jacinto RC, Zaia AA, Ferraz CCR (2013) Microbiological profile and antimicrobial susceptibility pattern of infected root canals associated with periapical abscesses. Eur J Clin Microbiol Infect Dis 32: 573-80.

Copyright: (C2016 Pfaff CE. This is an open-access article distributed under the terms of the Creative Commons Attribution License, which permits unrestricted use, distribution, and reproduction in any medium, provided the original author and source are credited. 